\title{
RESEARCH
}

Open Access

\section{Human adipose-derived stem cells enriched with VEGF-modified mRNA promote angiogenesis and long-term graft survival in a fat graft transplantation model}

Fei $\mathrm{Yu}^{1,2+}$, Nevin Witman ${ }^{3+}$, Dan Yan ${ }^{1,2}$, Siyi Zhang ${ }^{1,2}$, Meng Zhou ${ }^{1,2}$, Yan Yan ${ }^{1,2}$, Qinke Yao ${ }^{1,2}$, Feixue Ding ${ }^{4}$, Bingqian Yan ${ }^{5,6}$, Huijing Wang ${ }^{5,6}$, Wei Fu ${ }^{5,6^{*}}$, Yang $\mathrm{Lu}^{1,2^{*}}$ and $\mathrm{YaO} \mathrm{Fu}^{1,2^{*}}$ (D)

\begin{abstract}
Fat grafting, as a standard treatment for numerous soft tissue defects, remains unpredictable and techniquedependent. Human adipose-derived stem cells (hADSCs) are promising candidates for cell-assisted therapy to improve graft survival. As free-living fat requires nutritional and respiratory sources to thrive, insufficient and unstable vascularization still impedes hADSC-assisted therapy. Recently, cytotherapy combined with modified mRNA (modRNA) encoding vascular endothelial growth factor (VEGF) has been applied for the treatment of ischemiarelated diseases. Herein, we hypothesized that VEGF modRNA (modVEGF)-engineered hADSCs could robustly enhance fat survival in a fat graft transplantation model.

Methods: hADSCs were acquired from lipoaspiration and transfected with modRNAs. Transfection efficiency and expression kinetics of modRNAs in hADSCs were first evaluated in vitro. Next, we applied an in vivo Matrigel plug assay to assess the viability and angiogenic potential of modVEGF-engineered hADSCs at 1 week post-implantation. Finally, modVEGF-engineered hADSCs were co-transplanted with human fat in a murine model to analyze the survival rate, re-vascularization, proliferation, fibrosis, apoptosis, and necrosis of fat grafts over long-term follow-up.

\footnotetext{
* Correspondence: fuweizhulu@163.com; luyang8311@hotmail.com; drfuyao@126.com

${ }^{\dagger}$ Fei Yu and Nevin Witman contributed equally to this work.

${ }^{5}$ Department of Pediatric Cardiothoracic Surgery, Shanghai Children's Medical Center, School of Medicine, Shanghai Jiao Tong University, Shanghai 200127, China

'Department of Ophthalmology, Ninth People's Hospital, School of Medicine, Shanghai Jiao Tong University, Shanghai 200011, China

Full list of author information is available at the end of the article
}

(c) The Author(s). 2020 Open Access This article is licensed under a Creative Commons Attribution 4.0 International License, which permits use, sharing, adaptation, distribution and reproduction in any medium or format, as long as you give appropriate credit to the original author(s) and the source, provide a link to the Creative Commons licence, and indicate if changes were made. The images or other third party material in this article are included in the article's Creative Commons licence, unless indicated otherwise in a credit line to the material. If material is not included in the article's Creative Commons licence and your intended use is not permitted by statutory regulation or exceeds the permitted use, you will need to obtain permission directly from the copyright holder. To view a copy of this licence, visit http://creativecommons.org/licenses/by/4.0/ The Creative Commons Public Domain Dedication waiver (http://creativecommons.org/publicdomain/zero/1.0/) applies to the data made available in this article, unless otherwise stated in a credit line to the data. 


\begin{abstract}
(Continued from previous page)
Results: Transfections of modVEGF in hADSCs were highly tolerable as the modVEGF-engineered hADSCs facilitated burst-like protein production of VEGF in both our in vitro and in vivo models. modVEGF-engineered hADSCs induced increased levels of cellular proliferation and proangiogenesis when compared to untreated hADSCs in both ex vivo and in vivo assays. In a fat graft transplantation model, we provided evidence that modVEGF-engineered hADSCs promote the optimal potency to preserve adipocytes, especially in the long-term post-transplantation phase. Detailed histological analysis of fat grafts harvested at 15, 30, and 90 days following in vivo grafting suggested the release of VEGF protein from modVEGF-engineered hADSCs significantly improved neo-angiogenesis, vascular maturity, and cell proliferation. The modVEGF-engineered hADSCs also significantly mitigated the presence of fibrosis, apoptosis, and necrosis of grafts when compared to the control groups. Moreover, modVEGF-engineered hADSCs promoted graft survival and cell differentiation abilities, which also induced an increase in vessel formation and the number of surviving adipocytes after transplantation.
\end{abstract}

Conclusion: This current study demonstrates the employment of modVEGF-engineered hADSCs as an advanced alternative to the clinical treatment involving soft-tissue reconstruction and rejuvenation.

Keywords: hADSCs, modVEGF, Fat transplantation, Angiogenesis, Graft survival

\section{Background}

There is currently a clinical need for novel employable techniques to reconstruct congenital and acquired soft tissue defects in an efficient and safe manner. Fat grafting has become a preferred clinical treatment for soft-tissue augmentation and reconstruction, as it is a source of autologous cells and is easily obtained through liposuction procedures. Autologous fat is currently employed for a variety of cosmetic or reconstructive indications including sequelae of radiation therapy, breast augmentation, trauma, facial rejuvenation, and orbital fracture [1,2]. Despite the advantages of the wide sources of fat and its nonimmunogenic properties, the long-term poor outcomes of fat grafting caused by high absorption rate are critical drawbacks and a major limitation $[3,4]$. As reported, the survival rate of grafted fat ranges from 20 to $80 \%[5,6]$. The resulting volume reduction observed in fat grafts is met with adipocyte apoptosis and necrosis, fibrocystic invasion, and eventually loss of fat volume and function, which is mainly caused by insufficiency and untimely revascularization [7].

In this regard, numerous approaches have been applied to fat grafting procedures involving co-treatment regimes in the form of recombinant proteins, biomaterials, and gene modifications of stem cells in hopes to stimulate pro-angiogenesis/vascularization of the grafts $[8,9]$. The vast majority of these approaches have been met with contradictory outcomes. More recently, cellassisted fat transfer employing human adipose-derived stem cells (hADSCs) has been shown to significantly enhance fat graft survival [10,11]. As reported, hADSCs not only have the characteristics of rapid amplification, non-immunogenicity, and pluripotent differentiation potential, but also are easily and abundantly accessed via standard liposuction procedures $[12,13]$. However, the full potential of hADSCs in regenerative research has not yet been realized, as many researchers continue to engineer hADSCs to more effectively enhance aspects of regenerative medicine [14-16].

Synthetic chemically modified mRNAs (modRNAs) are gaining praise as a novel modality for forced, transient gene expression [17-19]. Unlike DNA and viral plasmids/vectors, modRNAs are expressed as potent and transient bursts of gene expression, capable of delivering an efficient and dose-controlled level of protein, while exhibiting minimal immunogenicity [17]. Several studies employing different myocardial infarction models revealed that intramyocardial injection of modRNA encoding the vascular endothelial growth factor $\mathrm{A}_{165}$ (VEGF- $\mathrm{A}_{165}$ ) gene-induced cardiac regeneration $[20,21]$. Moreover, a recent clinical study also suggested that intradermal injection of VEGF modRNA (modVEGF) was well tolerated in men with type 2 diabetes mellitus (T2DM), where the delivery induced local functional VEGF protein expression leading to increased dermal blood flow [22]. More recently, Yu et al. applied a cell-mediated modVEGF delivery system employing human fibroblasts to treat critical limb ischemia (CLI) [23]. In this aforementioned study by $\mathrm{Yu}$ et al., a cell-mediated approach for protein delivery through modRNAs revealed enhanced tissue regeneration when compared to the naked delivery of modVEGF alone by extending the window of expression and increasing the therapeutic potency of the protein, ultimately giving rise to healthier blood vessels. Worth mentioning, VEGF is expressed at subtle levels in hADSCs, where it is thought to play a role in increasing the survival of fat grafts [14, 24-26]. Taken together, we proposed that a stem cell-proangiogenic factor hybrid therapy might be beneficial in enhancing the angiogenic potential of hADSCs and could significantly improve fat retention post-transplantation. 
Herein, for the first time, we combined modVEGF with hADSCs from patient lipoaspirates to improve survival of fat transplantation. We report that, compared to hADSCs alone, modVEGF-engineered hADSCs more efficiently enhanced fat grafting through mechanisms involving neo-angiogenesis, cell proliferation, and simultaneously alleviated levels of fibrosis, apoptosis, and necrosis in the ischemic setting. Furthermore, modVEGF-engineered hADSCs were met with preserved viability and enhanced differentiation into vessels and adipocytes in situ. Thus, the combination of hADSCs with modRNAs could be an advanced course of action for regenerative therapies, including fat transplantation for tissue augmentation and reconstructive surgery.

\section{Materials and methods}

Isolation and culture of human adipose-derived stem cells (hADSCs)

The adipose tissue was derived from healthy female patients whom underwent liposuction or autologous fat transplantation for cosmetic purposes. The Ethics Committee of Shanghai Ninth People's Hospital approved the study. Standard liposuction techniques were employed as previously reported, and hADSCs were isolated and cultured following reported protocols [8, 27]. Briefly, fat tissue for grafting and hADSC extraction was separated from lipoaspirates via rinsing with PBS, centrifugation, and aspiration. The fat tissue was digested with $0.2 \%$ collagenase A (Roche, Manheim, Germany) at $37^{\circ} \mathrm{C}$ for $1 \mathrm{~h}$, followed by centrifugation at $1500 \mathrm{rpm}$ for $10 \mathrm{~min}$. The cell pellet was resuspended in Dulbecco's modified Eagle's medium (DMEM) (HyClone, GE Healthcare, Little Chalfont, UK) supplemented with $10 \%$ fetal bovine serum (FBS) (Invitrogen, California, USA) and 1\% penicillin-streptomycin antibiotic (Gibco, Carlsbad, CA, USA) and then seeded on $10-\mathrm{cm}$ dishes and subcultured using trypsin (Gibco, Carlsbad, CA, USA) when the cells reached $80-90 \%$ confluence. hADSCs were employed between passages 3 and 5 for all studies described.

\section{modRNA synthesis and formulation}

modRNA was synthesized in vitro using T7 RNA polymerase-mediated transcription from a linearized DNA template, which incorporated generic $5^{\prime}$ and $3^{\prime}$ UTRs and a poly-A tail, as previously described [23, 28, 29]. During the in vitro transcription reaction, we fully replaced uridine with N1-methylpseudouridine. RNA was purified using Ambion MEGAclear spin columns and treated with Antarctic phosphatase (New England Biolabs) for $30 \mathrm{~min}$ at $37^{\circ} \mathrm{C}$ to remove residual $5^{\prime}$-phosphates. The RNA was repurified and quantified by Nanodrop (Thermo Scientific, Waltham, MA, USA), and then, modRNA was resuspended in $10 \mathrm{mM}$ Tris $\mathrm{HCl}, 1$
mM EDTA at $1 \mu \mathrm{g} / \mu \mathrm{L}$ for use. Open reading frame sequences used for modRNA production were the same as previously described for EGFP, firefly Luciferase, and human VEGF-A 165 [20].

\section{modRNA transfection}

In order to transfect hADSCs with modRNAs, MessengerMAX (Invitrogen, California, USA) transfection reagent was employed. For in vitro experiments, modRNAs and transfection reagents were first diluted separately in OptiMEM basal medium (Invitrogen, California, USA) and incubated for $5 \mathrm{~min}$ at room temperature (RT). Afterwards, the two mixes were pooled together and incubated for 15 min to generate modRNA-lipid complexes. modRNAlipid complexes were exposed to cells for $4 \mathrm{~h}$, after which the medium was completely replaced with cell culture media or removed to collect cells. Specifically, for lipocomplexing, $2 \mu \mathrm{L}$ of MessengerMAX transfection reagent was used per $1 \mu \mathrm{g}$ modRNA to transfect per $1 \times 10^{5}$ hADSCs in the in vitro and in vivo experiments.

For evaluating GFP modRNA (modGFP) expression kinetics in hADSCs, the transfection efficiency and mean fluorescence intensities of modGFP were recorded at 4 , $8,16,24$, and $48 \mathrm{~h}$ post-transfection, by C6 flow cytometry (Beckman Coulter, CA, USA).

\section{Expression of modVEGF in vitro Real-time PCR}

Total RNA from cultured hADSCs were extracted by TRIzol reagent (Invitrogen, California, USA) at $24 \mathrm{~h}$ following transfection, and the reverse transcription was employed using PrimeScript RT reagent kit (Takara Bio Inc., Otsu, Japan) as previously reported [14]. Real-time PCR was performed using Power SYBR Green PCR Master Mix (Applied Biosystems, Foster, CA, USA) according to the protocol. The following primers were used: VEGF-A, forward primer, 5'-TTGCAGGTTGGTT CCCAGAGG-3' and reverse primer, 5' -TCGGCTTGTC ACATCTGAGGG-3'; $\beta$-actin, forward primer, $5^{\prime}$ GGGACCTGACTGACTACCTC-3'; and reverse primer, 5'-TCAT ACTCCTGCTTGCTGAT-3'. qPCR detection was carried out on a 7500 Real-Time PCR Detection System (Thermo Scientific, Waltham, MA, USA), and relative expression was determined using the $2-\Delta \Delta C \mathrm{~T}$ method.

\section{Western blot assay}

Western blot analysis was conducted as previously described [30]. Briefly, total protein from cell lysates was extracted using lysis buffer (Thermo Scientific, Waltham, MA, USA) and the concentration was measured using a BCA kit (Pierce, Rockford, IL, USA). A 50-mg protein was loaded onto SDS-PAGE and then transferred to polyvinylidene difluoride membrane. After blocking with 5\% 
skimmed milk, the membranes were then incubated with primary antibodies against VEGF-A (ab52917; Abcam, MA, USA) and $\beta$-actin (ab8226; Abcam, MA, USA) at $4{ }^{\circ} \mathrm{C}$ overnight. After incubation with DyLightTM680conjugated secondary antibodies (Sigma, St. Louis, MO, USA), the protein expression levels were quantified using an Odyssey V 3.0 image scanner (LI-COR, Lincoln, Nebr., USA).

\section{Enzyme-linked immunosorbent assay (ELISA)}

For measuring the expression kinetics of modVEGF, the cell culture supernatant was collected at specified time points $(4,8,16,24,48,72,96,120 \mathrm{~h}$ after transfection) and concentrations of human VEGF-A protein were quantified using ELISA (R\&D Systems, Inc., Minneapolis, MN, USA) according to the manufacturer's instructions. The optical density values of absorbance were measured on a microplate reader (ELX800, BioTek, USA).

\section{Characteristics of hADSCs Proliferation of hADSCs}

hADSCs were seeded in a 96-well plate at a concentration of $2 \times 10^{3}$ cells/well, each group containing six counter holes. After the treatments, the Cell Counting Kit-8 (CCK8) (Dojindo, Kumamoto, Japan) was used to conduct a cell proliferation assay at different time points. An optical density at $450 \mathrm{~nm}$ was recorded using a microplate reader (ELX800, BioTek, USA). Average data were presented as percentages of the O.D. values relative to the control group.

\section{Migration of hADSCS}

Migration assay was performed using 24-well polycarbonate membrane cell culture plate inserts with $8-\mu \mathrm{m}$ pore size (SPL Life Sciences Co., Ltd., Korea). At first, hADSCs in each group were cultured for $24 \mathrm{~h}$ posttransfection. After trypsinization, $2 \times 10^{4}$ cells were seeded on the top chambers of the transwell inserts. The top chambers contained $200 \mu \mathrm{L}$ serum-free medium, and the bottom chambers contained $400-\mu \mathrm{L}$ of $10 \%$ serum medium. After $12 \mathrm{~h}$ of incubation, cells passed through the membrane were dyed by crystal violet and counted to calculate the means of triplicate experiments.

\section{Phenotypic profile of hADSCS}

The phenotypic profile of hADSCs was determined by flow cytometry as previously described [31]. Briefly, approximately $5 \times 10^{4}$ cells were incubated with fluorescence-conjugated antibodies, which were all obtained from BD Biosciences for $30 \mathrm{~min}$ at RT. Quantitative analysis was performed using C6 flow cytometry (Beckman Coulter, CA, USA), and results were analyzed by FlowJo software (Tree Star Inc., Ashland, OR, USA).

\section{Multipotent differentiation of hADSCS}

The trilineage differentiation potentials of hADSCs were tested as performed in a previous article [32]. All chemicals were purchased from Sigma, St. Louis, MO, USA. According to instructions, pretreated-hADSCs were cultured separately in AdipoDiff, OsteoDiff, and ChondroDiff Medium for 21 days, followed by Oil Red O, Alizarin Red S, and Alcian Blue stainings. Images of differentiated hADSCs were obtained and evaluated.

\section{Matrigel plug assay}

Six-week-old male BALB/c-nude mice in our study were purchased from the Animal Laboratory, Shanghai Ninth People's Hospital, Shanghai Jiao Tong University School of Medicine, Shanghai, China. All experimental protocols were approved by the Animal Care and Experiment Committee of the Shanghai Ninth People's Hospital. Three mice were used for the implantation of Matrigel plugs as previously described [23]. In brief, ice-cold Matrigel (BD Biosciences, San Jose, CA, USA) $(200 \mu \mathrm{L}$ per plug) was mixed with $50 \mu \mathrm{L}$ PBS or hADSCs $\left(1 \times 10^{6}\right.$ cells in $50 \mu \mathrm{L}$ PBS) and then injected subcutaneously into the dorsal region of mice (each mouse received three plugs in total from the different groups). The plugs of the PBS group contained Matrigel matrix mixed with only PBS; the plugs containing Matrigel matrix mixed with hADSCs pretreated with either Luciferase modRNA (modLuc) or modVEGF were termed the ADSC ${ }^{\text {modLuc }}$ group or the ADSC ${ }^{\text {modVEGF }}$ group, respectively. For the ADSC $^{\text {modLuc }}$ and ADSC ${ }^{\text {modVEGF }}$ groups, a total of $10 \mu \mathrm{g}$ modRNA and $20 \mu \mathrm{L}$ MessengerMAX reagent was used for $1 \times 10^{6}$ cells. One week after implantation, Matrigel plugs were excised for gross and histological evaluation.

\section{Fat transplantation model}

Nude mice models of fat grafting were constructed as previously described [33]. The mice were randomly divided into the PBS group, the $\mathrm{ADSC}^{\text {modLuc }}$ group and the ADSC $^{\text {modVEGF }}$ group. In the PBS group, a mixture of $400 \mu \mathrm{L}$ of fat with $100 \mu \mathrm{L}$ of PBS alone was injected. In the $\mathrm{ADSC}^{\text {modLuc }}$ group, a mixture of $400 \mu \mathrm{L}$ of fat with $100 \mu \mathrm{L}$ of PBS containing $2 \times 10^{6}$ modLuc-engineered hADSCs was injected. In ADSC ${ }^{\text {modVEGF }}$ group, a mixture of $400 \mu \mathrm{L}$ of fat with $100 \mu \mathrm{L}$ of PBS containing $2 \times 10^{6}$ modVEGF-pretreated hADSCs was injected. Before injection, the mixture of each group was mixed well in dishes and syringes of each group were not cross-used. Thirty-six mice in total were used. As shown in Fig. 4a, each mouse received implants of three fat grafts via a 14-G needle, where each graft represented a different treatment group. Fat grafts were harvested at desired time points for further analysis. We randomly selected eighteen mice for sacrifice at 1, 2, 3, 4, 5, and 6 days after grafting to evaluate in vivo protein levels of VEGF-A 
and HIF1 $\alpha$ by ELISA (R\&D Systems, Inc., Minneapolis, MN, USA) ( $n=3$ each time point). The remaining eighteen mice were sacrificed at 15,30 , and 90 days posttransplantation, and the fat grafts were photographed, weighed, and underwent further histological evaluation ( $n=6$ each time point). Additionally, three mice sacrificed at 90 days received fat grafts mixed with modLuc or modVEGF-engineered hADSCs that were first subjected to CM-DiI labeling (Invitrogen, California, USA). $\mathrm{CM}$-DiI labels were performed as the instruction described.

\section{Histological staining}

The Matrigel plugs and fat grafts were paraffinembedded and cut into 6- $\mu \mathrm{m}$-thick sections. Hematoxylin \& eosin (H\&E) staining was performed, and fat vacuoles, identifying the adipocytes larger than $120 \mu \mathrm{m}$ in diameter, were evaluated as previously described [34]. To evaluate collagen accumulation, indicating fibrosis, Masson trichrome (Sigma, St. Louis, MO, USA) stainings were performed according to the manufacturer's instructions.

For immunohistological staining, tissue sections were probed with primary antibodies at $4{ }^{\circ} \mathrm{C}$ overnight, followed by incubation with a $\mathrm{Cy}^{\mathrm{mm}} 3$-conjugated secondary antibody (Jackson ImmunoResearch Laboratories, Inc., PA, USA) or Alexa Fluor 488-conjugated secondary antibodies (Invitrogen, California, USA). The following is a list of the primary antibodies used for immunohistochemistry: anti-VEGF-A antibody (ab52917; Abcam, MA, USA), anti-CD31 antibody (ab182981; Abcam, MA, USA), anti-Human Nuclear Antigen (ab191181; Abcam, MA, USA), anti-Perilipin antibody (ab61682; Abcam, MA, USA), anti- $\alpha$-SMA antibody (ab21027; Abcam, MA, USA), anti-Ki67 antibody (ab92742; Abcam, MA, USA), and anti-TNF- $\alpha$ antibody (ab1793; Abcam, MA, USA). Terminal deoxynucleotidyl transferase-mediated d-UTP nick end labeling (TUNEL) (Roche, Manheim, Germany) staining was performed following the manufacturer's instructions. Photomicrographs were performed using a confocal microscope (Leica microsystems, Heidelberg, Germany). Five random fields were selected for each sample ( $n=3$ each time point). All measurements were performed with Image-Pro Plus software (Media Cybernetics, MD, USA).

\section{Statistical analysis}

The data are presented as the mean \pm standard deviation. $p$ values were analyzed using a one-way analysis of variance (ANOVA) followed by Tukey's test (GraphPad Software, San Diego, CA, USA). Statistical significance is denoted by $p<0.05$.

\section{Results}

Transfection of hADSCs with modified mRNAs (modRNAs) are well tolerated

In order to determine the transfection efficiency and kinetics of modRNAs in hADSCs, we transfected hADSCs with modRNA encoding a GFP reporter construct (modGFP). We found that hADSCs were highly tolerant of modRNA transfections as we demonstrated the transfection efficiency of modGFP in hADSCs as high as $92.2 \% \pm 2.7 \%$ at $16 \mathrm{~h}$ post-transfection (Fig. 1a, b and Figure S1). The highest mean fluorescence intensity signal of the GFP protein was recorded at $2 \times 10^{6}$ at $24 \mathrm{~h}$ after transfection (Fig. 1c).

In order to confirm the transfection efficiency and expression dynamics of modRNAs in hADSCs, we again separately transfected hADSCs with modRNAs encoding either the Luciferase (modLuc) or VEGF- $\mathrm{A}_{165}$ (modVEGF) genes and monitored transcript levels inside the cells. RTPCR revealed more than 70,000-fold increase of VEGF mRNA expression in the modVEGF-engineered hADSC (ADSC ${ }^{\text {modVEGF}}$ ) group at $24 \mathrm{~h}$ after transfection, in comparison to the modLuc-engineered hADSCs (ADSC $^{\text {mo- }}$ ${ }_{\text {dLuc }}$ group (Fig. 1d). Using western blot analysis, we demonstrated that the intracellular levels of VEGF protein in the $\mathrm{ADSC}^{\text {modVEGF }}$ group expressed two-fold more VEGF protein $24 \mathrm{~h}$ after transfection than the two control groups, confirming the translation of the modRNA (Fig. 1e, f). Moreover, VEGF mRNA and protein levels did not differ between the untransfected (ADSC) group and the ADSC $^{\text {modLuc }}$ group (Fig. 1d-f).

As VEGF is a secreted ligand, we sought to detect the levels of newly produced and accumulated VEGF protein levels in the cell culture media following transfection in hADSCs. We found that VEGF protein concentrations were significantly higher in the medium of the ADSC $^{\text {modVEGF }}$ group from 4 to $144 \mathrm{~h}$ post-transfection when compared to the two control groups (Fig. 1g, h).

\section{Characteristics of hADSCs transfected with modRNAs}

In order to evaluate the effects of modVEGF on hADSCs, we characterized the levels of cell proliferation, migration, and differentiation capabilities post-transfection. Using the CCK8 assay, we showed that modVEGF could promote the proliferation of hADSCs to some extent (Fig. 2a). However, transwell assays revealed no differences in migration capability between the three groups (Fig. 2b, c). By evaluating the marked surface expression of stromal markers, we discovered that hADSCs maintained their "stemness" at $24 \mathrm{~h}$ after modRNA-transfection. As identified by the Mesenchymal and Stem Cell Committee of the International Society for Cellular Therapy (ISCT), our treated and untreated hADSCs positively expressed stromal markers CD73, CD90, and CD105 and negatively expressed hematopoietic and endothelial markers CD20, CD31, and CD45 (Figure S2 and Fig. 2d) 
A

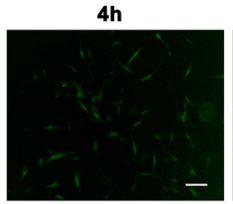

$8 h$
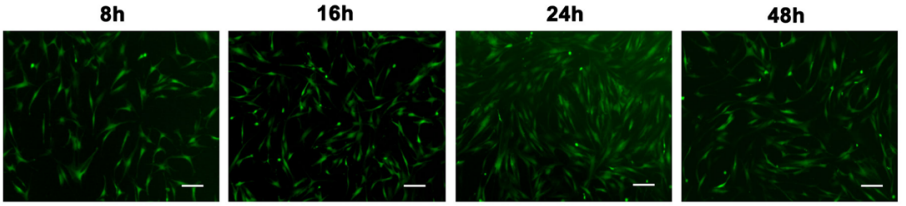

B

C
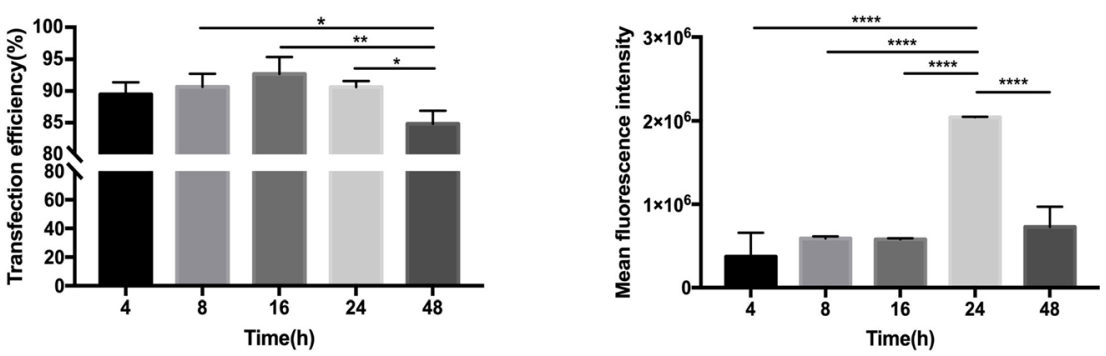

D

E

F
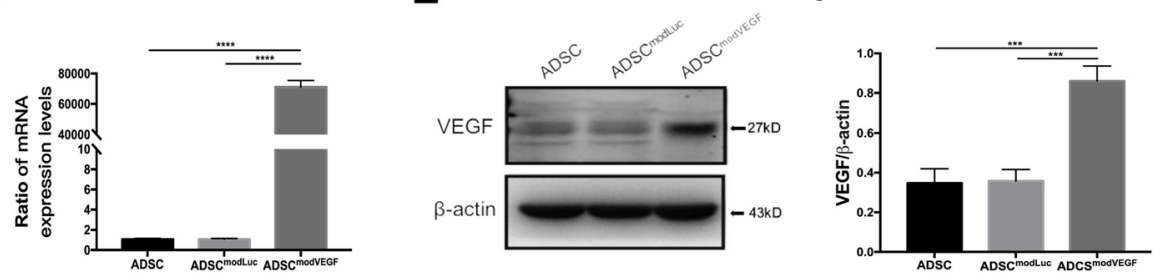

G

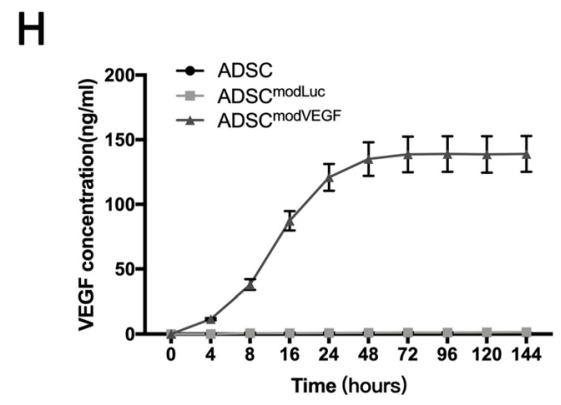

Fig. 1 Efficacy and kinetics of modRNA transfection in hADSCs. a-c Transfection efficiency and the expression kinetics of modGFP in hADSCs. a Representative images depicting GFP signal in hADSCs at 4, 8, 16, 24, and $48 \mathrm{~h}$ post-transfection. b Flow cytometry analysis of transfection efficiency at 4, 8, 16, 24, and $48 \mathrm{~h}$ post-transfection. c Flow cytometry analysis of mean fluorescence signal intensity at 4, 8, $\mathbf{1 6}, 24$, and $48 \mathrm{~h}$ post-transfection. $\mathbf{d}-\mathbf{f}$ Expression levels of $\mathbf{d}$ VEGF mRNA and $\mathbf{e}-\mathbf{f}$ VEGF protein at $24 \mathrm{~h}$ post-transfection. $\mathbf{g}-\mathbf{h}$ Kinetics of $\mathbf{g}$ newly produced and $\mathbf{h}$ cumulative VEGF protein concentrations periodically monitored for several days following transfection of modVEGF in hADSCs. Scale bar $=100 \mu \mathrm{m}$. Error bars showed means \pm SD. $\left(n=3 ;{ }^{*} p t<0.05,{ }^{* *} p+<0.01,{ }^{* * *} p+<0.001,{ }^{* * *} p \dagger<0.0001\right)$

[35]. Furthermore, hADSCs exhibit multipotent plasticity in vitro. Therefore, we employed a trilineage differentiation assay, which revealed that modRNA treatments did not negatively inhibit/alter the adipogenic, osteogenic, and/or chondrogenic differentiation capacities of the hADSCs (Fig. 2e).

\section{Confirming functional in vitro secretion of VEGF protein from modVEGF-engineered hADSCs}

In order to ensure that the VEGF protein secreted by modVEGF-engineered hADSCs was functionally active, the conditioned medium was applied to human umbilical cord vein cells (HUVECs). We quantitated levels of HUVEC proliferation and migration, as well as tube formation when cultured in hADSCmodVEGF conditioned media. We showed conditioned medium from modVEGF-engineered hADSCs could improve cell proliferation and migration more significantly than conditioned medium from hADSCs transfected with modLuc (Figure S3A-E). Based on a tube formation assay, we revealed more tubular structures were formed and significantly more junctions 


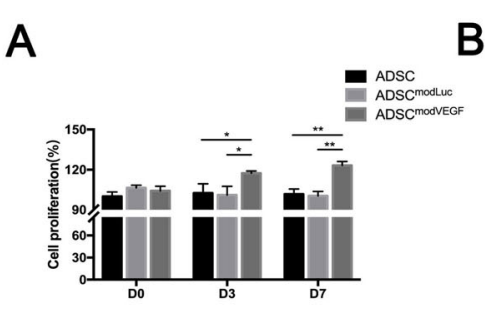

B

C

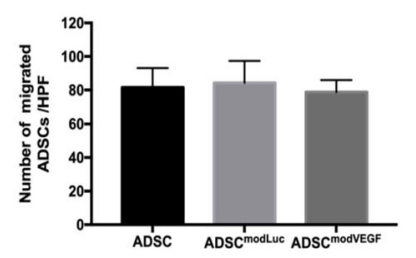

D

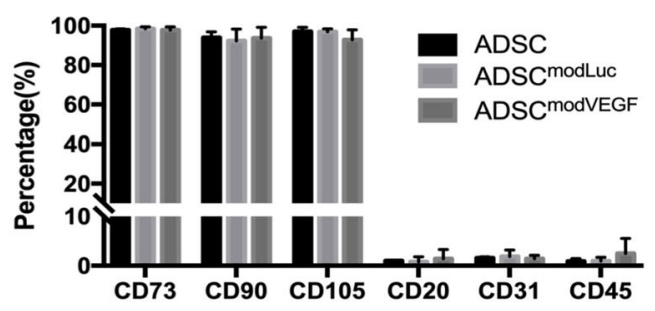

E

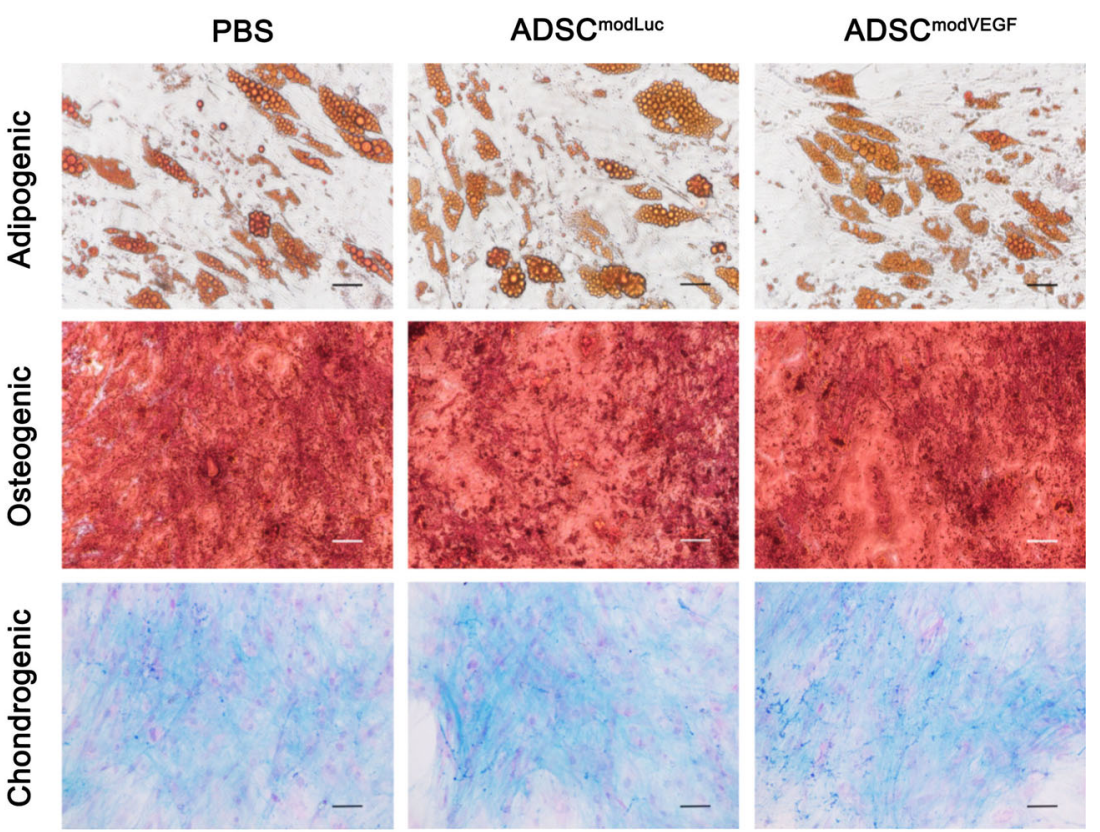

Fig. 2 Multipotent differentiation potential and characterization of isolated and mRNA-engineered hADSCs in vitro. a The proliferation capability of hADSCs was assessed using cell count kit, and the percentage of optical density values relative to the control was calculated. $\left(n=6\right.$; ${ }^{*} p+<0.05$, ** p广<0.01). b, c The migration potential of hADSCs was evaluated using transwell assay, and the number of migrated cells dyed with crystal violet (at $12 \mathrm{~h}$ post-seeding) was quantified. $\mathbf{d}$ Cell surface marker expression of hADSCs was analyzed at $24 \mathrm{~h}$ post-transfection. e The multipotent capacity of hADSCs following modRNA transfections was tested by inducing adipogenic, osteogenic, and chondrogenic differentiations. Successful differentiations of the lineages were detected and analyzed using Oil Red O, von Kossa, and Alcian Blue stainings, respectively. Scale bars $=50 \mu \mathrm{m}$. Error bars showed means $\pm \mathrm{SD} .\left(n=3 ;{ }^{*} p+<0.05\right)$

had formed in HUVECs cultured in the ADSC ${ }^{\text {modVEGF }}$ conditioned media (Figure S3F-H). Interestingly, subtle increases in the endothelial migration and proliferation of HUVECs were seen in the ADSC ${ }^{\text {modLuc }}$ group when compared to the control group. As hADSCs secrete low levels of chemokines and growth factors that enhance certain aspects of cell growth and proliferation, as previously reported, this finding was not completely unexpected (Figure S3) [26]. Since modLuc is inert but did not hinder beneficial effects seen from the hADSCs, we employed this treatment group as a continued control throughout our study. 
Proangiogenic, anti-apoptotic, and pro-proliferative effects of modVEGF-engineered hADSCs in an in vivo Matrigel plug assay

In order to further explore the potential pro-angiogenic, pro-proliferative, and anti-apoptotic properties of hADSCs transfected with modVEGF post-transplantation, we employed an in vivo Matrigel plug model. To examine the angiogenic efficacy of transfected modVEGF in hADSCs, we compared Matrigel mixtures containing modVEGFengineered hADSCs (ADSC ${ }^{\text {modVEGF}}$ ) against Matrigel mixtures containing modLuc-engineered hADSCs $\left(\right.$ ADSC $\left.{ }^{\text {modLuc }}\right)$. We also included Matrigel mixtures with PBS as a negative control (PBS) group. By employing this assay, we sought to directly visualize if modVEGFengineered hADSCs could induce new blood vessel formation in vivo. Matrigel mixtures were implanted into the subcutaneous space of the dorsal region in mice and were harvested after 1 week post-implantation for gross morphological observation (Fig. 3a) and histological analysis (Fig. 3b-j). By evaluating initial gross morphology, the plugs of the $\mathrm{ADSC}^{\text {modLuc }}$ group and the ADSC ${ }^{\text {modVEGF }}$ group both appeared more vascularized than the PBS group (Fig. 3a). Furthermore, the plugs in the ADSC ${ }^{\text {mod- }}$ VEGF group appeared significantly more vascularized and the plugs in whole appeared more morphologically complete when compared to the $\mathrm{ADSC}^{\text {modLuc }}$ group (Fig. 3a). Notably, we discovered that the Matrigel complexes containing ADSC $^{\text {modVEGF }}$ gave rise to more abundant VEGF protein than $\operatorname{ADSC}^{\text {modLuc }}(p<0.01)$ even at 1 week post-transplantation (Fig. 3b, c). In Fig. 3d, histological analysis revealed the spatial distribution of human nuclei (HNA) and vascular endothelial cells (CD31), where new blood vessels appeared to be in close proximity to the hADSCs. Following quantification, the number of hADSCs in the ADSC ${ }^{\text {modVEGF }}$ group was significantly higher than those found in the PBS and hADSC ${ }^{\text {modLuc }}$ groups (Fig. 3e). Quantitative assessment of $\mathrm{CD} 31^{+}$stainings revealed that plugs mixed with $\mathrm{ADSC}^{\text {modVEGF }}$ formed significantly more capillaries than hADSC ${ }^{\text {modLuc }}(p<$ 0.0001 ) (Fig. 3f). The ADSC ${ }^{\text {modVEGF }}$ group induced better angiogenic activity from intrinsic tissue than the $\mathrm{ADSC}^{\mathrm{mo}-}$ dLuc group, probably due to the increased number of surviving hADSCs and more potent levels of secreted VEGF protein. In order to monitor cell viability in vivo, the levels of proliferation and cell death were analyzed by employing anti-ki67 stainings and TUNEL assays (Fig. $3 g-j$ ). Plugs harvested from the hADSC ${ }^{\text {modVEGF }}$ group presented with significantly more $\mathrm{Ki}^{+} 7^{+}$cells when compared to the ADSC $^{\text {modLuc }}$ group $(p<0.05)$, indicating the modVEGF treatment enhanced cell proliferation rates of hADSCs within the plugs (Fig. 3g, h). There were also significantly fewer apoptotic cells in the ADSC ${ }^{\text {modVEGF }}$ group than in the PBS $(p<0.05)$ and the ADSC ${ }^{\text {modLuc }}$ groups, respectively (Fig. 3i, j). Taken together, these results confirm that
modVEGF-engineered hADSCs better enhanced the viability of implanted hADSCs leading to better angiogenic, pro-proliferative, and anti-apoptotic activities than hADSCs alone.

\section{modVEGF-engineered hADSCs significantly improve fat graft survival in vivo}

In order to examine the active effects of modRNAengineered hADSCs on fat transplantation, the human fat was transplanted into nude mice together with PBS, modLuc-engineered hADSCs or modVEGF-engineered hADSCs (Fig. 4a). To determine the in vivo produced protein levels of VEGF expression stemming from modRNA-engineered hADSCs and non-treated hADSCs in fat grafts, we extracted and purified total protein from the fat implants at $1,2,3,4,5$, and 6 days posttransplantation. Interestingly, we found expression levels of in vivo produced human VEGF protein in the ADSC $^{\text {modVEGF }}$ group to be superiorly significant, nearly $5000 \mathrm{ng} / \mathrm{g}$ protein within the first $24 \mathrm{~h}$ post transplantation, as opposed to concentrations under $150 \mathrm{ng} / \mathrm{g}$ protein in the other groups $(p<0.0001)$ (Fig. 4b). These extremely high levels of VEGF protein production were continually detectable within the first $48 \mathrm{~h}$ after transplantation in the ADSC ${ }^{\text {modVEGF }}$ group (Fig. 4b). On the 3rd day, VEGF protein levels from the ADSC ${ }^{\text {modVEGF }}$ transplant group subsided; however, the protein levels remained significantly higher than the control groups (Fig. 4b). On the 4th day post-transplantation and the remainder of the quantified period (days 5 and 6 posttransplant), the levels of detected VEGF protein were similar between both the ADSC $^{\text {modLuc }}$ and ADSC $^{\text {mod- }}$ VEGF groups, which remained significantly higher than the PBS only control group (Fig. 4b). To confirm the role of the VEGF pathway further and in order to define the functional efficacy of VEGF protein in fat grafts, we examined the concentration of HIF $1 \alpha$ protein. HIF $1 \alpha$ protein is an upstream regulator of VEGF, whose expression levels are known to represent the degree of hypoxia in fat grafts $[8,36]$. We quantified the expression levels of HIF $1 \alpha$ across our treatment groups and controls. HIF1 $\alpha$ levels were significantly reduced in the ADSC ${ }^{\text {mod- }}$ VEGF group during days $2-4$ post-implantation, but most notably on the 4th day post-grafting $(p<0.001)$ (Fig. 4c). Taken together, these findings suggest that modVEGFengineered hADSCs vastly enhanced therapeutic protein levels and impacted biological activities in order to regulate hypoxia-conditioning in fat grafts in vivo.

To further confirm the functional properties of modVEGF-engineered hADSCs on fat transplantation, we analyzed the gross morphology and coarse weight of grafts at 15,30 , and 90 days post-transplantation $(n=6$ each time point). Representative images of grafts within each group are shown in Fig. 4d. The weights of fat 


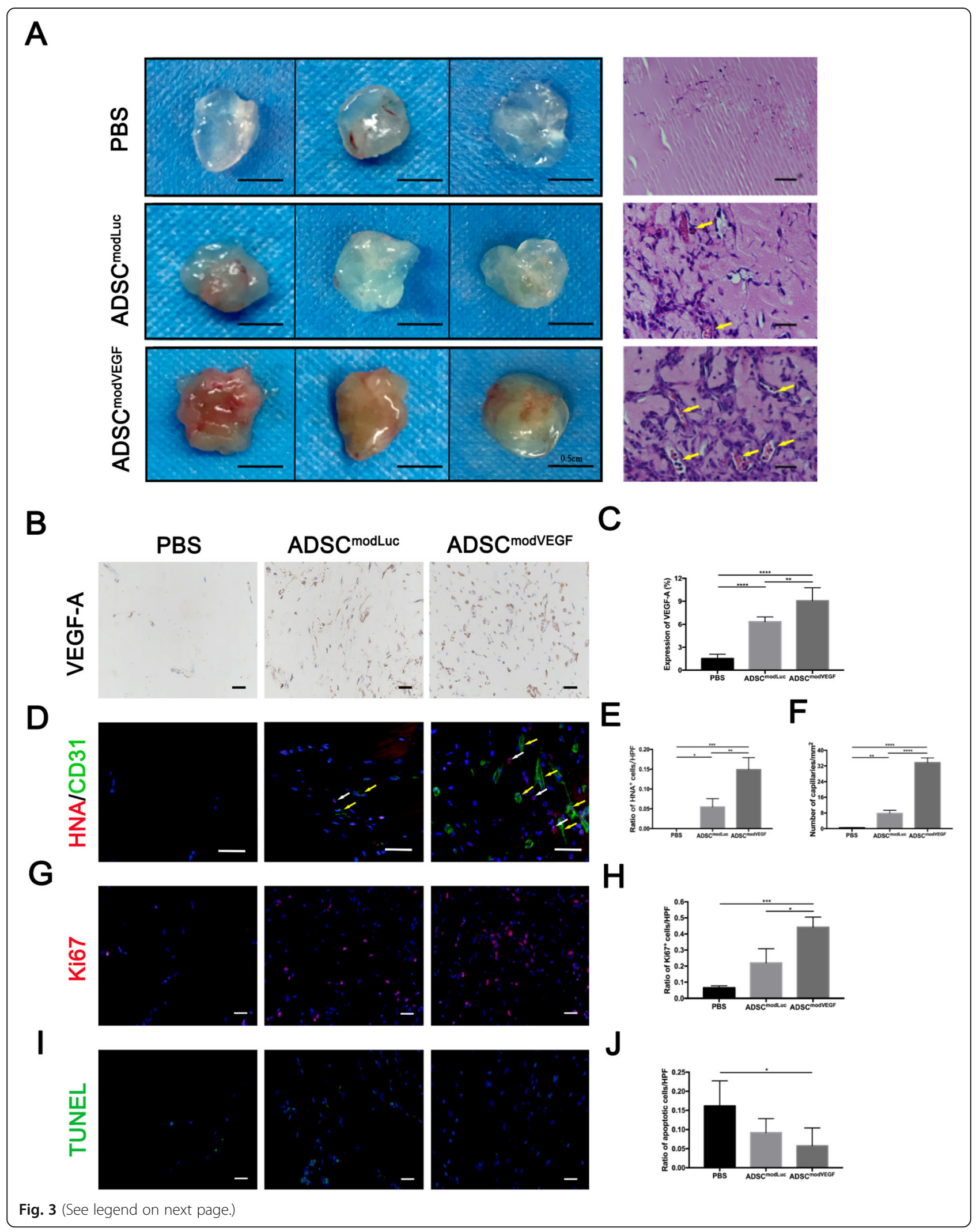


(See figure on previous page.)

Fig. 3 hADSCs engineered with modVEGF promotes angiogenesis in an in vivo Matrigel plug assay. Matrigel plugs harvested at 1-week post-implantation were assessed. a Representative gross morphological assessment (left) and microphotographs (right) of hematoxylin \& eosin (H\&E) stainings of extracted plugs. Yellow arrows indicate new blood vessel formation within the plug. b Representative photomicrographs of Matrigel plugs stained for VEGF detection. c The percentage of VEGF expression per area was calculated. $\mathbf{d}$ Representative photomicrographs of Matrigel plugs stained for human nuclei antibody (HNA) and CD31. White arrows partially indicate the localization of hADSCs detected with HNA. Yellow arrows partially indicate CD31+ blood vessels. e, $\mathbf{f}$ Ratio of HNA ${ }^{+}$ cells (e) and the number of $\mathrm{CD} 31^{+}$capillaries were quantified (f). $\mathbf{g}$ Representative images depicting levels of proliferating hADSCs in plugs using Ki67 stainings. $\mathbf{h}$ The ratio of Ki67 $7^{+}$cells was quantified. $\mathbf{i}$ Representative TUNEL stainings depict levels of apoptotic hADSCs within the Matrigel plugs. $\mathbf{j}$ The ratio of apoptotic cells was quantified. Error bars showed means \pm SD. Scale bar $=20 \mu \mathrm{m} .\left(n=3,{ }^{*} p t<0.05,{ }^{* *} p t<0.01,{ }^{* * *} p t<0.001,{ }^{* * * *} p+<0.0001\right)$

grafts in the ADSC ${ }^{\text {modVEGF }}$ group were significantly higher than those in the PBS group at all investigated time points (Fig. $4 \mathrm{e}-\mathrm{g}$ ) and differences between the two cell-assisted fat transplant groups became evident after 2 weeks post-grafting (on the 15th day) (Fig. 4e). On the 30th day, the effects of cell-assisted fat transplants became more prominent, where significant differences were observed between both the ADSC $^{\text {modLuc }}$ and ADSC $^{\text {modVEGF }}$ groups when compared to PBS control (Fig. 4f). Of note, the weights of the fat grafts in the ADSC $^{\text {modVEGF }}$ group at 90 days post-transplantation were $0.283 \pm 0.024 \mathrm{~g}$, which were significantly higher $(p<0.05)$ than $0.241 \pm 0.018 \mathrm{~g}$ in the ADSC ${ }^{\text {modLuc }}$ group (Fig. 4g). Taken together, these results suggest that fusing modRNA technologies with stem cell-assisted fat transplants could help enhance the protective functions of hADSCs, thus further promoting heightened grafted fat survival, especially over the long term.

\section{Transplantation of modVEGF-engineered hADSCs enhances morphology and viability of adipocytes after grafting}

To more deeply examine the protective potential of modVEGF-engineered hADSCs in our fat transplantation model, fat grafts were also harvested for histological studies at days 15, 30, and 90 posttransplantation. H\&E stainings revealed a significant reduction in quantifiable vacuoles and connective tissue forming from the hADSC ${ }^{\text {modVEGF }}$ treatment group when compared to the PBS and ADSC ${ }^{\text {modLuc }}$ groups (Fig. 5a). Furthermore, both cell-assisted fat transplant groups, namely the $\mathrm{ADSC}^{\text {modVEGF }}$ as well as the $\mathrm{ADSC}^{\text {modLuc }}$ group revealed better anatomical morphology of adipose tissue than the PBS group, where the ADSC ${ }^{\text {modVEGF }}$ group gave rise to the least amount of adipocyte vacuoles (Fig. 5b). The reserved functional adipocytes of all grafts were quantitated by perilipin stainings. The morphology and area (\%) of perilipin ${ }^{+}$adipocytes indicated that fat grafts in the ADSC ${ }^{\text {modVEGF }}$ group retained significant viability over the control groups throughout the observed timeline and the differences among the three groups became apparent over time (Fig. 5c, d). From these observations, we infer that modVEGFengineered hADSCs had the optimal effects on the survival of adipocytes and the advantages of VEGFsecreting hADSCs became gradually significant.

\section{modVEGF-engineered hADSCs enhance angiogenesis and} proliferative activities in fat grafts in vivo

To further evaluate the status of the grafted fat tissue, which had been treated with modVEGF-engineered hADSCs, we harvested and stained the grafts at 15, 30, and 90 days post-transplantation to assess neovessel formation and levels of cell proliferation. Using anti-CD31 antibodies and performing staining and analysis, we found more capillaries were formed in both the hADSC cell-assisted fat graft treatment groups, as visible already at day 15 post-transplantation (Fig. 6a). Note that the effects from the ADSC ${ }^{\text {modVEGF }}$ group gave rise to the most abundant capillary densities, which was evident in stained sections (Fig. 6a). In addition, when compared to the $\mathrm{ADSC}^{\text {modLuc }}$ group, the $\mathrm{ADSC} \mathrm{modVEGF}^{\text {group pre- }}$ sented with significantly larger vessel diameters and higher capillary densities, which together indicated better nutrient supply to adipocytes, at 90 days after grafting $(p<0.05)$ (Fig. 6b, c). Moreover, by analyzing percentages of proliferating cells in the grafts following transplantation, we found that both hADSCs and modVEGFengineered hADSCs significantly promoted proliferation over the PBS control group. However, the ADSC ${ }^{\text {modVEGF }}$ group presented with the highest levels of cell proliferation at all time points studied (Fig. 6d, e). These findings suggest that transplantation of modRNA-engineered hADSCs induced neo-angiogenesis and increased cellular proliferation at levels significantly higher than those stemming from the non-modified hADSC-assisted fat transplant group. These findings suggest that the fusion of this molecular therapy together with cell-assisted fat grafts enhanced the composition of the grafted tissue in vivo.

\section{modVEGF-engineered hADSCs reduce the long-term absorption rate of grafted fat via alleviating fibrosis, apoptosis, and necrosis}

As the hADSC ${ }^{\text {modVEGF }}$ group presented with prominent beneficial effects, we next wanted to further investigate the long-term stability of these fat grafts at 90 days following transplantation. Morphological assessment of the 


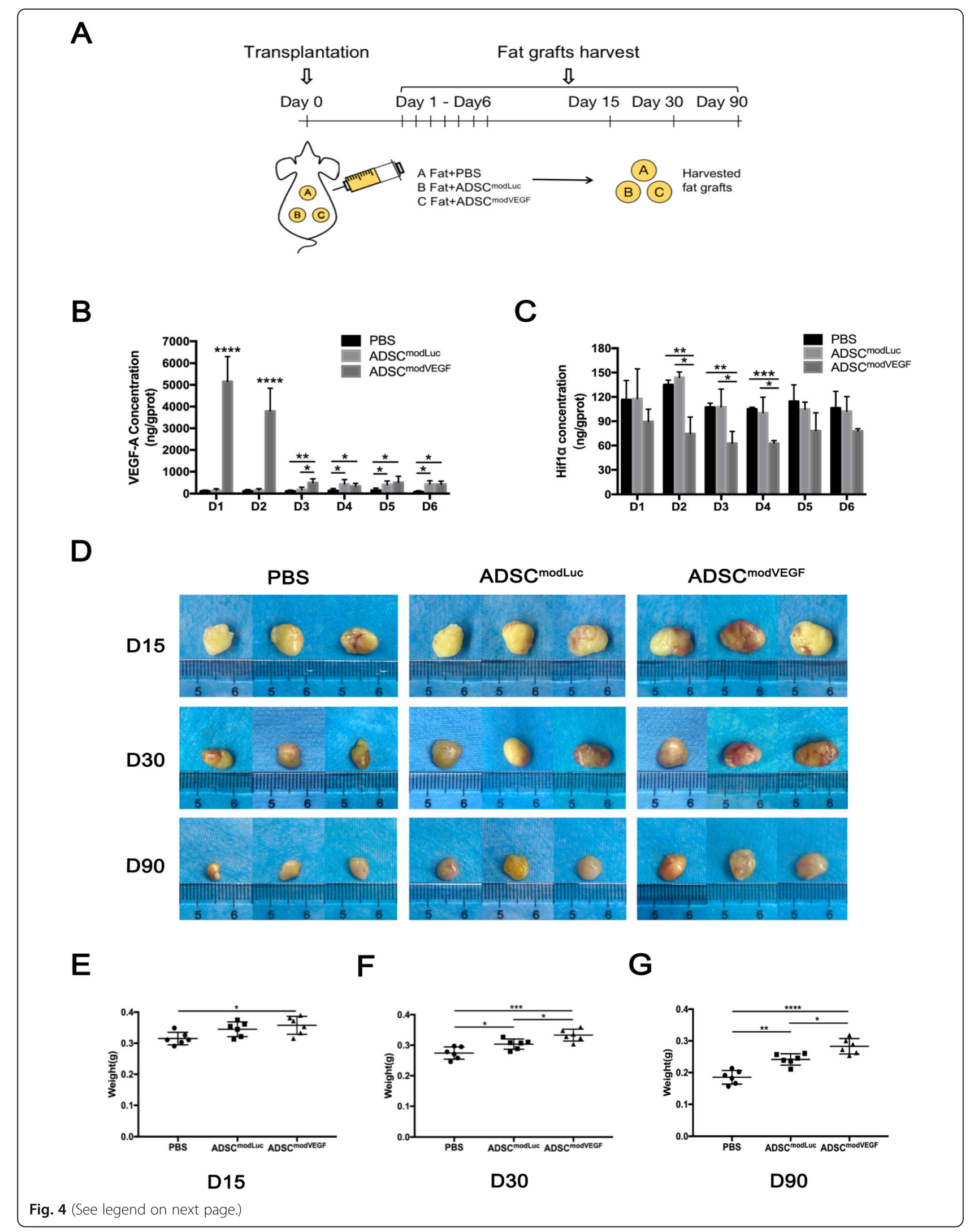


(See figure on previous page.)

Fig. 4 Preconditioning hADSCS with modVEGF improves fat graft survival in vivo. a Schematic illustration and design of the animal study. $\mathbf{b}$, $\mathbf{c}$ Quantification of $\mathbf{b}$ VEGF-A in vivo produced protein and $\mathbf{c}$ HIF1a protein levels in fat grafts at days 1, 2, 3, 4, 5, and 6 post-transplantation. ( $n=3$ ) d Representative gross morphological images of fat grafts at 15, 30, and 90 days post-transplantation. e-g Weights of the fat grafts at 15, 30, and 90 days post-transplantation. $(n=6)$ Error bars showed means \pm SD. $\left({ }^{*} p t<0.05,{ }^{* *} p \dagger<0.01,{ }^{* * *} p \dagger<0.001,{ }^{* * * *} p \dagger<0.0001\right)$

grafts using Masson's trichrome staining and analysis revealed that the $\mathrm{ADSC}^{\text {modVEGF }}$ group presented with significantly less fibrotic tissue among the three treatment groups, which is quantified in Fig. 7a, b $(p<0.001)$. The percentages of $\alpha-\mathrm{SMA}^{+} \mathrm{CD} 31^{-}$cells represent the fibrotic adipocytes (Fig. 7c, d). The ADSC ${ }^{\text {modVEGF }}$ group maintained the healthiest looking adipose tissue, as blood vessels generated from this group were predominantly $\alpha$ $\mathrm{SMA}^{+} \mathrm{CD} 31^{+}$, demonstrating the ability for this treatment group to form more mature vessels and less fibrotic cells (Fig. 7c, e). TUNEL stainings revealed that there was a significant difference in levels of apoptosis between the $\mathrm{ADSC}^{\text {modLuc }}$ and the ADSC ${ }^{\text {modVEGF }}$ groups $(p<0.05)$ (Fig. 7f, g). Moreover, the ADSC ${ }^{\text {modVEGF }}$ group, but not the ADSC ${ }^{\text {modLuc }}$ group had significantly lower ischemia-induced necrosis when compared to the PBS group, as depicted by TNF- $\alpha$ levels $(p<0.05)$ (Fig. 7h, i). hADSCs engineered with modVEGF were capable of attenuating ischemia-induced fibrosis, apoptosis, and necrosis over a long-term follow-up and as such enhanced fat graft survival.

\section{modVEGF-engineered hADSCs enhance viability, retention, and in vivo differentiation to support a long- term survival of fat grafts}

In order to trace and explore the fate of modRNAengineered hADSCs, we employed CM-DiI labeling and histologically assessed fat grafts after a long-term transplantation in vivo. Simple histological analysis revealed more CM-Dil-labeled hADSCs had survived in the ADSC $^{\text {modVEGF }}$ group at 90 days following grafting (Fig. 8a, b) $(p<0.05)$ when compared to the ADSC ${ }^{\text {modLuc }}$ control group. To determine the detailed fates of hADSCs, we stained sectioned grafts with a green fluorescein-labeled CD31 or perilipin to distinguish vascular endothelial cells and adipocytes derived from the exogenous CM-DiI-labeled hADSCs (Fig. 8c-f). Merging of the red fluorescence of CM-DiI with the green fluorescence of CD31 revealed a large portion of hADSCs treated with modVEGF took on an endothelial cell fate (Fig. 8c, d) $(p<0.05)$. Furthermore, an increased quantification of yellow adipocytes labeled with $\mathrm{CM}-\mathrm{DiI}^{+}$and green fluorescein-Perilipin ${ }^{+}$was detected in the $\mathrm{ADSC}^{\text {modVEGF }}$ group, indicating better induction to mature adipocytes (Fig. 8e, f) $(p<0.01)$. Together, these results indicate hADSCs treated with modVEGF mRNA can better maturate into adipocytes and vascular endothelial cells, which ultimately enhance the longterm retention of transplants.

\section{Discussion}

Herein, we report the first study emphasizing the therapeutic potential of combining hADSCs with modRNA technologies in order to reduce absorption rates in a humanized fat transplantation model.

At the early stage of fat grafting, there is no blood supply in grafts and the only source of nutrients is limited to the plasma surrounding the grafts [7]. As reported, timely and adequate revascularization is crucial for the survival of fat grafts, particularly in patients whom undergo large-volume fat transplants $[37,38]$. Over the years, many investigators have reported the promise of hADSCs for cytotherapy via enhancing angiogenesis. hADSCs are a type of mesenchymal stromal/stem cell (MSC) arising from adipose tissue; meanwhile, other sources of MSCs include those isolated from the bone marrow (BMSCs), umbilical cord blood (UCB-MSCs), muscle (MDSCs), and dental pulp (DPSCs) [13]. Compared to the other populations of MSCs, several key advantages to working with hADSCs include the ease of isolation with minimal donor discomfort, as well as a higher quantity of collected cells (i.e., approx. 500-fold more MSCs are obtained in fat as opposed to BMSCs) $[39,40]$. It has also been shown that hADSCs have more robust differentiation capabilities and as such may be better suited for treating a wider spectrum of ischemic injuries [33, 40-46]. Therefore, hADSCs have been exploited as a biological tool for angiogenic therapy, especially for tissue reconstruction, combating ischemiarelated diseases, wound healing, and immune-regulation [26, 47-49].

Although ADSC-assisted fat transplants have been regarded as an optimal option to promote graft survival, previous studies observed hADSCs undergoing cell death prematurely and thus failed to induce timely and adequate angiogenesis post-transplantation [50-52]. VEGF, as a naturally secreted paracrine factor of hADSCs, directly promotes angiogenesis; however, one could speculate that the low levels of endogenously produced and secreted VEGF in these cells are insubstantial for a longterm vascularization [53, 54]. Fusing hADSC biologics with other cell-free-based technologies, such as recombinant human (rh) proteins or DNA vectors, are worthy alternatives to more effectively induce a long-term and stable angiogenesis. In our studies, we did not pursue 


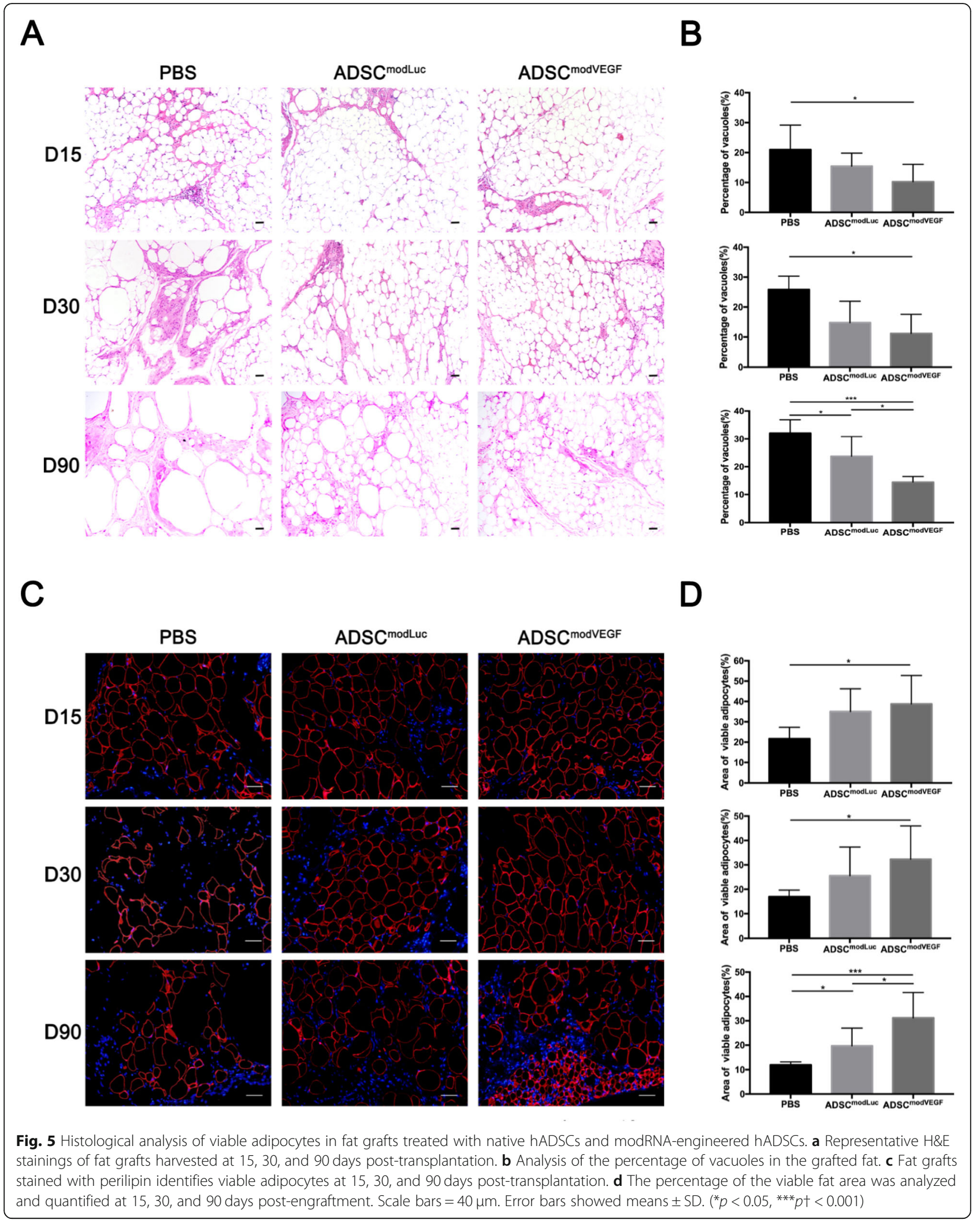




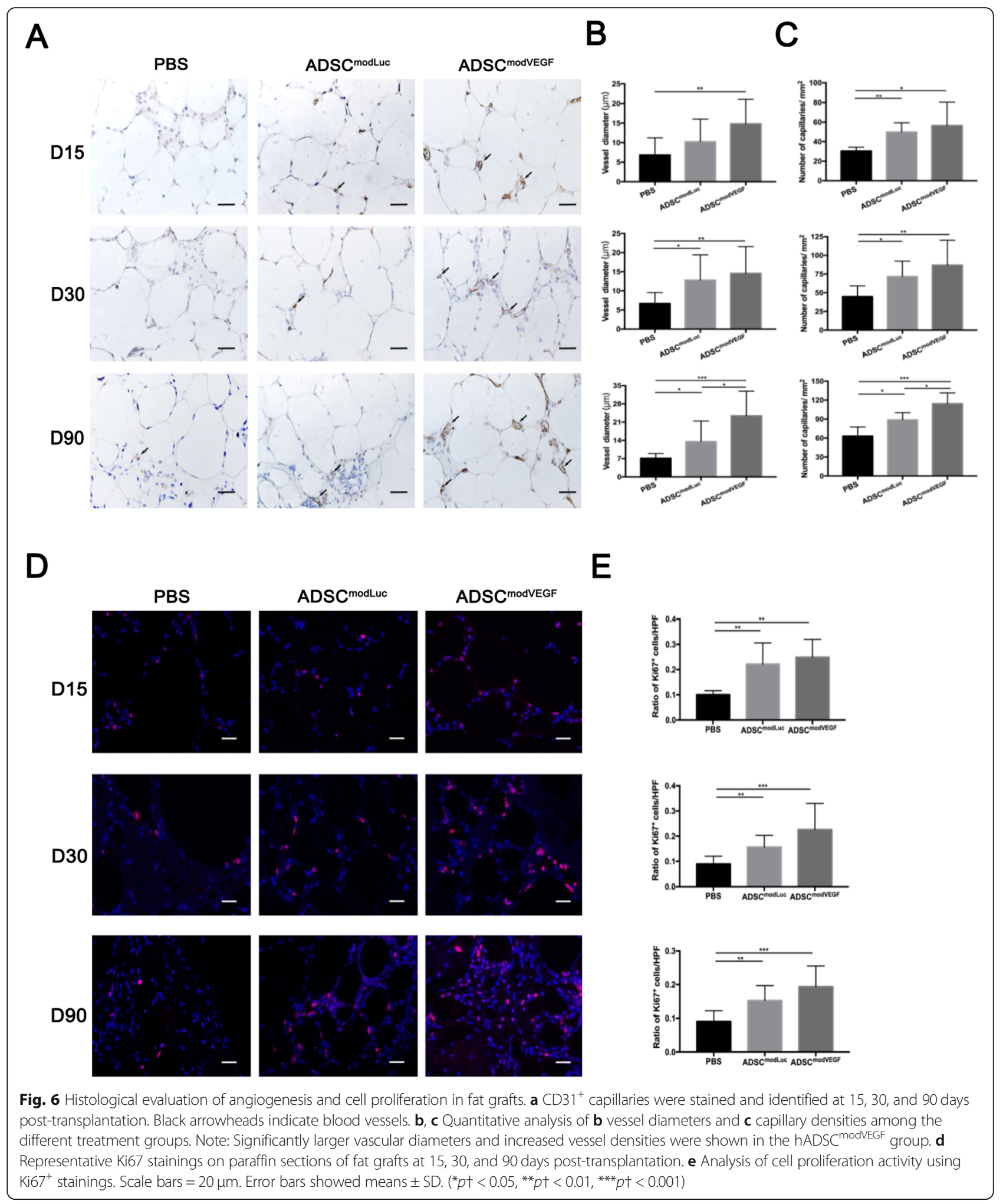

such activities as VEGF rh proteins are used at supraphysiological doses to circumvent their short half-life, the results of which have led to the formation of leaky blood vessels and negative side-effects $[20,55]$. On the other hand, DNA-based methods to engineer VEGFsecreting hADSCs would induce genomic integration and force long-term expression, a potential risk for systemic safety $[16,56]$. Intriguingly, the significant 


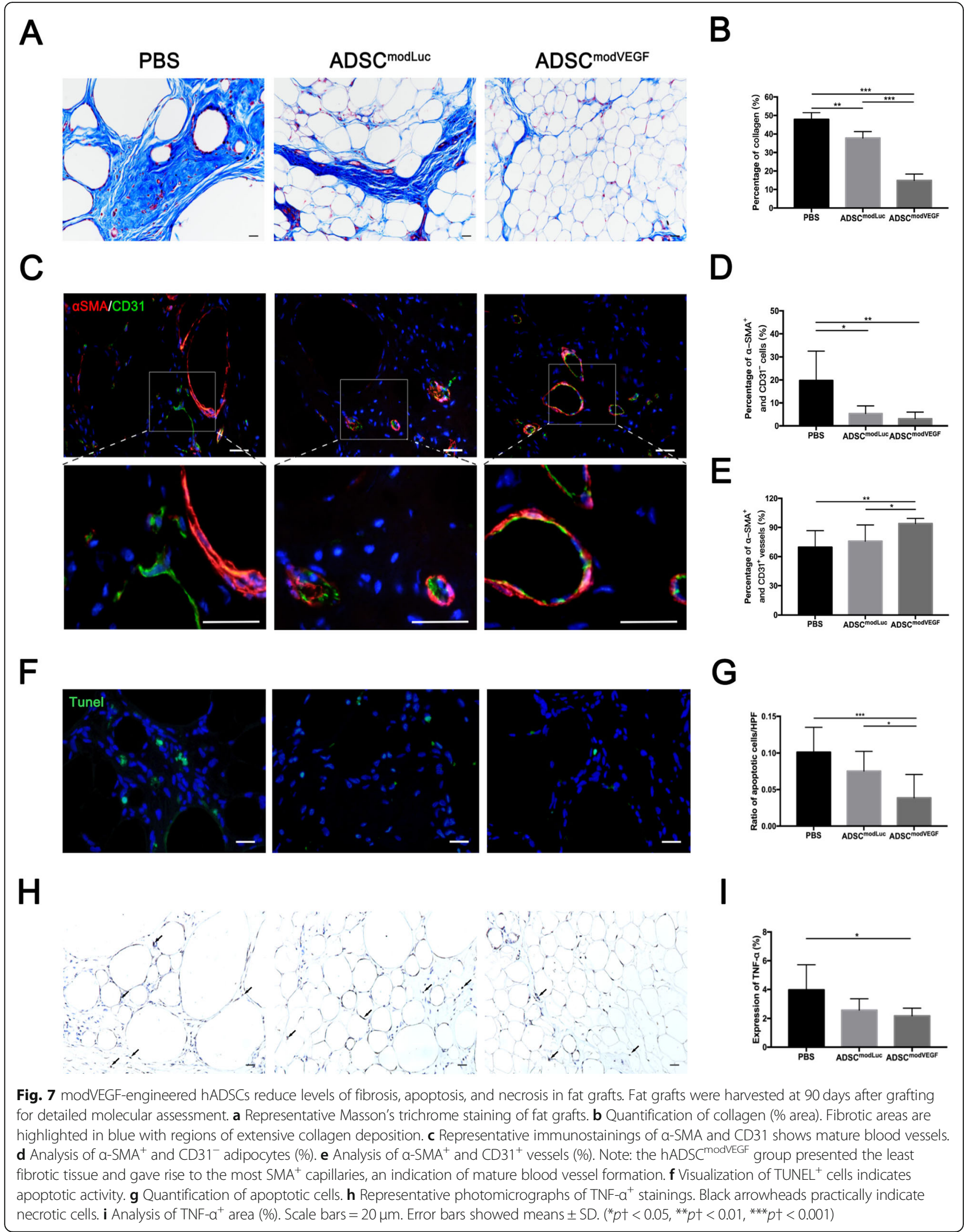




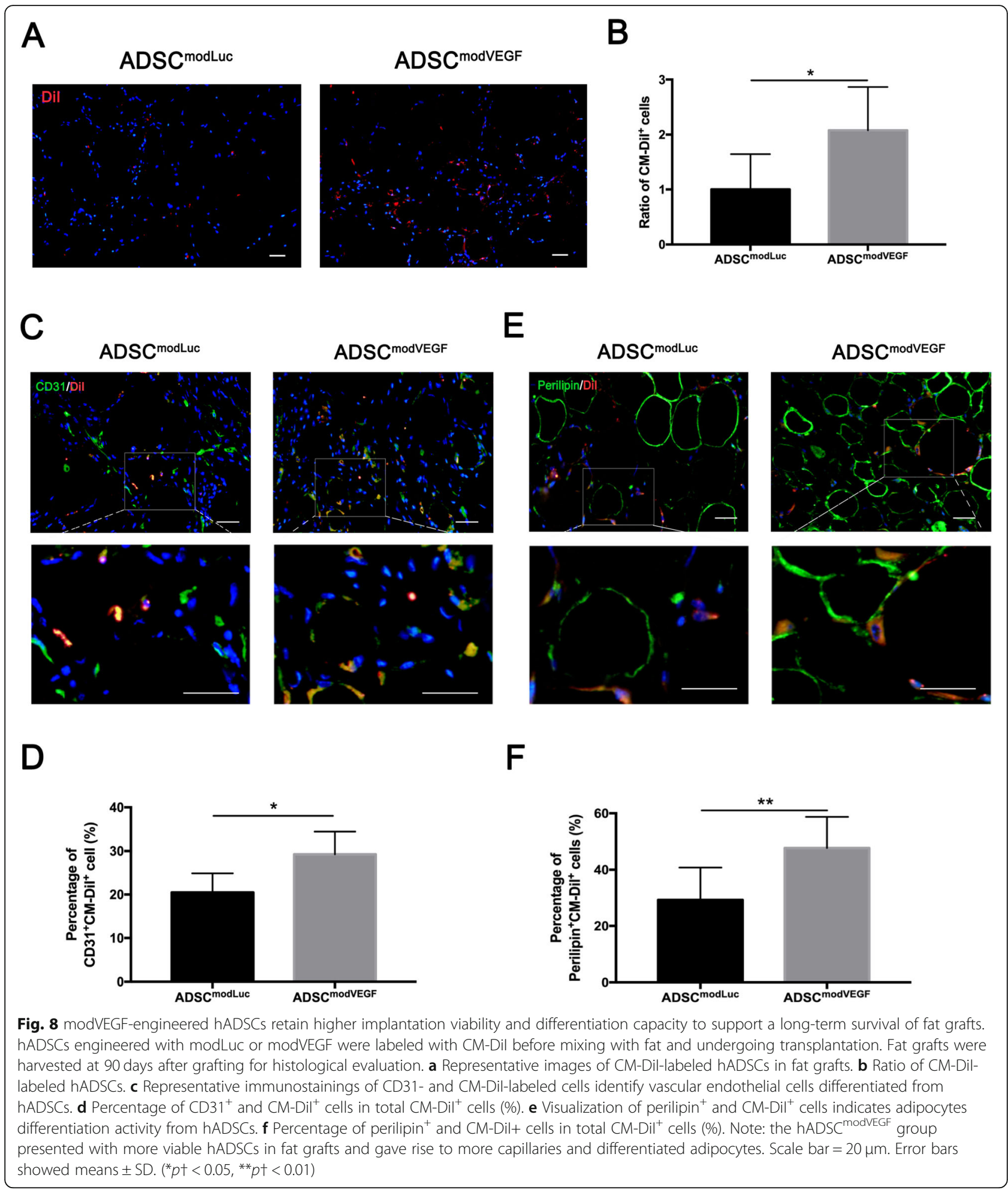

advantages of a modRNA-based therapeutic modality are many, as highlighted in previous reports [17-20]. For example, the delivery and expression kinetics of modRNAs are met with high biocompatibility and low immunogenicity (Figs. 1and 2) [20, 23]. In addition,
modRNA constructs are synthetically derived and can therefore be produced quickly and in large-scale format for applied and pre-clinical research in animal models of disease $[57,58]$. Furthermore, the forced expression of VEGF protein from modRNA-engineered cells can 
exhibit burst-like therapeutic expression levels of VEGF protein in vivo before the loss of transplanted cells caused by initial ischemia, which has been reported (Fig. $1 \mathrm{~g}$ and $4 \mathrm{~b})[23,56]$.

Previous research reported that viable adipocytes of grafted fat were dramatically decreased as early as $24 \mathrm{~h}$ after transplantation, due to ischemic damage [7]. In our study, we found that modVEGF-engineered hADSCs were less prone to ischemic injury through mechanisms involving adequate vascularization and relieved hypoxic conditions (Fig. $1 \mathrm{~h}$ and Fig. 4b, c). The transient yet abundant VEGF-A protein secreted from the modVEGFengineered hADSCs was found to not only directly contribute to enhanced viability of hADSCs (Fig. 2a), but also increase the capacity for a long-term survival and better promote the in situ cell-fate potential (Fig. 3d, e and Fig. 8). We further revealed that the modVEGFengineered hADSCs could stimulate neovascularization in a more favorable manner and induce the retention of more viable grafted cells enhancing the regenerate as made evident by the presence of expanded adipocytes (Fig. 5). Moreover, the ADSC ${ }^{\text {modVEGF }}$ group gave rise to significant vascularization and cell proliferation, seemingly persistent at 90 days post-transplantation (Fig. 6). In addition, modVEGF-engineered hADSCs could alleviate cell fibrosis, apoptosis, and necrosis over a long-term follow-up post-transplantation, which provides a strong case for the employment of hADSCs combined with
modRNA technologies in the treatment of ischemic diseases (Fig. 7). Furthermore, previous studies have highlighted VEGF protein acting as a critical modulator of the tissue fibrosis and enhanced VEGF secretion potentially preventing/limiting apoptosis and necrosis under hypoxic conditions by activating the PI3K/Akt, TGF- $\beta$, or Notch pathways [59-61]. In this regard, it would be of interest to further evaluate mechanisms of modVEGF-engineered hADSCs on graft survival in our model. Exosomes isolated from hADSCs in hypoxic culture have also been reported to provoke a positive response regarding the survival of grafts and enhancing angiogenic ability [15]. Whether or not MSCs engineered with modRNA could enhance the beneficial effects seen by applications with exosomes is a worthy outlet worth exploring.

Consistent with reported studies, we found no malignancy resulting from the hADSCs or modVEGFengineered hADSCs applied in this study [10, 22]. Additionally, we provide novel support for effective therapies of modVEGF and broadened the application of modRNAs to tissue transplantation. As indicated in Fig. 9, we believe our therapeutic approach provides an optimal alternative to stem cell-assisted treatment in tissue transplantation and in regenerative medicine. In theory, stem cells could be easily harvested, carefully manipulated with modRNAs, and applied to the ischemic tissue to preserve cellular function and promote survival. Due
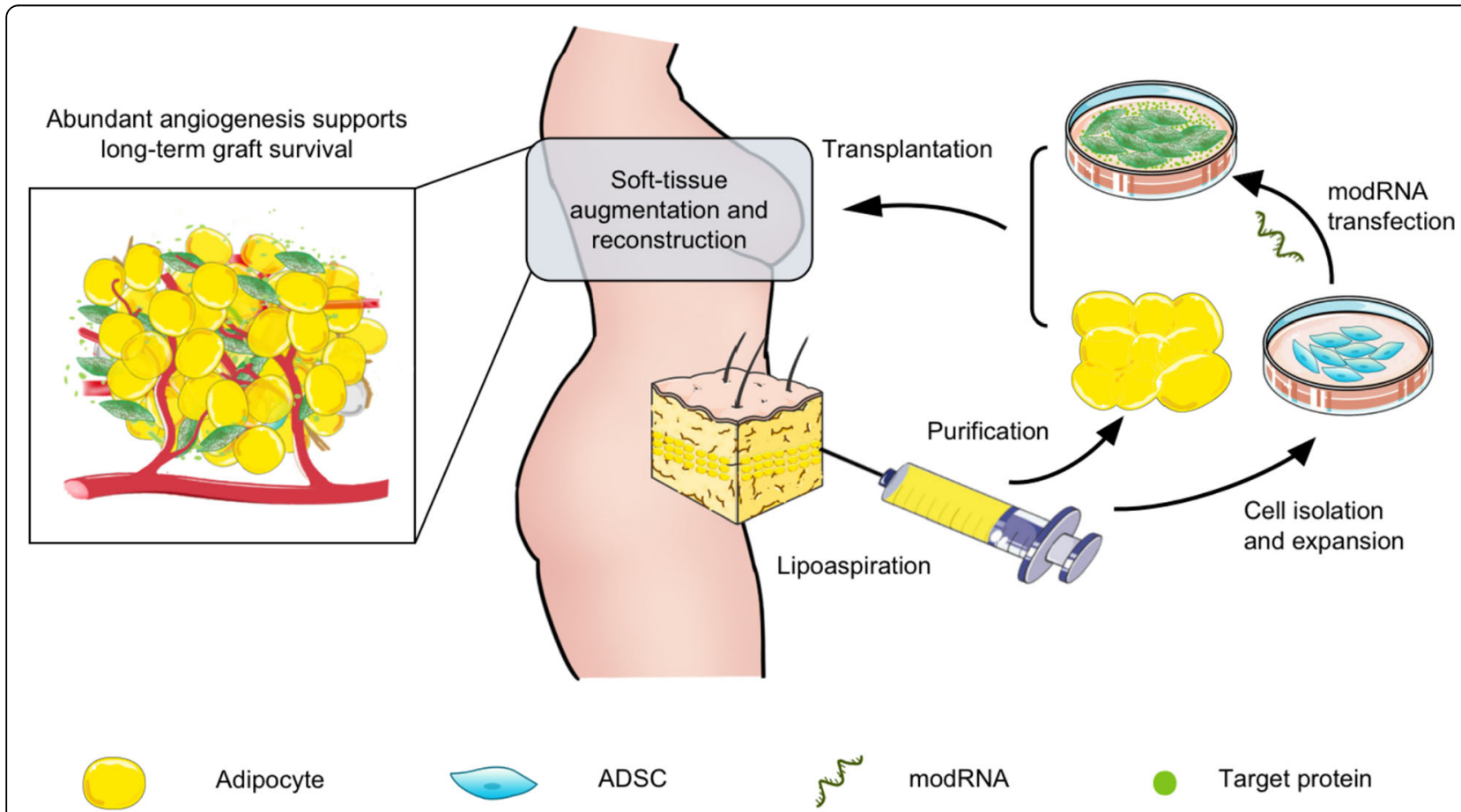

Target protein

Fig. 9 A novel combined model of hADSCs engineered with modRNAs could be considered as a potential treatment to promote angiogenesis and graft survival for clinical soft-tissue augmentation and reconstruction 
to the safety and effectiveness of hADSCs and modRNAs, the novel combined model of hADSCs engineered with modRNAs could be considered as a potential treatment in the clinic for grafting and ischemic injuries.

\section{Conclusion}

Our study highlights the optimal therapeutic potential of stem cells combined with modRNA technologies in tissue grafting. Importantly, results stemming from our study indicate that combining modRNA technologies with hADSCs dramatically enhances the therapeutic activity of the cells. Particularly, when applied to fat grafting, modVEGF-engineered hADSCs significantly improved the retention of fat grafts over naíve hADSCs through proangiogenic and pro-proliferative responses. Furthermore, this combinatorial treatment of hADSCs with modVEGF negatively regulated levels of fibrosis, apoptosis, and necrosis in fat grafts. This concept of a combinatorial treatment approach that merges cell-based and cell-free therapies has great clinical promise and could be a therapeutic strategy for treating wound defects, injuries stemming from vascular damage, or autoimmune disorders such as Graft-Versus-Host-Disease (GVHD).

\section{Supplementary Information}

The online version contains supplementary material available at https://doi. org/10.1186/s13287-020-02008-8.

\section{Additional file 1}

\section{Abbreviations}

hADSCs: Human adipose-derived stem cells; modRNA: Modified mRNA; VEGF-A: Vascular endothelial growth factor A; modVEGF: VEGF-modified mRNA; modLuc: Luciferase-modified mRNA; MSCs: Mesenchymal stromal/ stem cells; BMSCs: Bone marrow-derived MSCs; T2DM: Type 2 diabetes mellitus; CLI: Critical limb ischemia; HUVECs: Human umbilical cord vein cells; ISCT: International Society for Cellular Therapy; H\&E: Hematoxylin \& eosin; TUNEL: Terminal deoxynucleotidyl transferase-mediated dUTP nick endlabeling; HIF-1a: Hypoxia-inducible factor 1-alpha; TNFa: Tumor necrosis factor alpha; aSMA: Alpha smooth muscle actin

\section{Acknowledgements}

Not applicable.

\section{Authors' contributions}

F.Y.: conception and design, collection and assembly of data, data analysis and interpretation, manuscript writing, and final approval of the manuscript. D.Y., S.Z., Y.Y., M.Z., F.D., and B.Y.: collection and assembly of data and final approval of the manuscript. H.W. and Q.Y.: data analysis and final approval of the manuscript. W.F., N.W., Y.L., and Y.F.: conception and design, data analysis and interpretation, manuscript writing, and final approval of the manuscript. The authors read and approved the final manuscript.

\section{Funding}

This work was supported by the National Natural Science Foundation of China (81570812, 81770888, 31800809, 81800873), Shanghai Municipal Education Commission-Gaofeng Clinical Medicine Grant Support (20161421), Shanghai Natural Science Foundation (20ZR1434500), Shanghai Pujiang Program (18PJD031), Shanghai Collaborative Innovation Center for Translational Medicine (TM201821), the Science and Technology Commission of Shanghai
(17DZ2260100), and fund form Shanghai Key Laboratory of Tissue Engineering.

Availability of data and materials

The datasets used and/or analyzed during the current study are available from the corresponding author on a reasonable request

\section{Ethics approval and consent to participate}

The present study was approved by the Ethics Committee of Shanghai Ninth People's Hospital and the Animal Care and Experiment Committee of Shanghai Ninth People's Hospital. Informed consent was obtained from all participants, and experiments were conducted along the guidelines set by the Ethics Committee of the Shanghai Ninth People's Hospital.

\section{Consent for publication}

Not applicable.

\section{Competing interests}

The authors indicated no potential conflicts of interest.

\section{Author details}

'Department of Ophthalmology, Ninth People's Hospital, School of Medicine, Shanghai Jiao Tong University, Shanghai 200011, China. ${ }^{2}$ Shanghai Key Laboratory of Orbital Diseases and Ocular Oncology, Shanghai 200011, China. ${ }^{3}$ Department of Cell and Molecular Biology, Karolinska Institute, Stockholm, Sweden. ${ }^{4}$ Department of Plastic Surgery, Ninth People's Hospital, School of Medicine, Shanghai Jiao Tong University, Shanghai 200011, China.

${ }^{5}$ Department of Pediatric Cardiothoracic Surgery, Shanghai Children's Medical Center, School of Medicine, Shanghai Jiao Tong University, Shanghai 200127, China. 'Institute of Pediatric Translational Medicine, Shanghai Children's Medical Center, School of Medicine, Shanghai Jiao Tong University, Shanghai 200127, China.

Received: 29 July 2020 Accepted: 3 November 2020

Published online: 19 November 2020

\section{References}

1. Simonacci F, Bertozzi N, Grieco MP, Grignaffini E, Raposio E. Autologous fat transplantation for breast reconstruction: a literature review. Ann Med Surg (Lond). 2016;12:94-100.

2. Borrelli MR, Patel RA, Sokol J, Nguyen D, Momeni A, Longaker MT, et al. Fat chance: the rejuvenation of irradiated skin. Plast Reconstr Surg Glob Open. 2019;7(2):e2092.

3. Tocco I, Widgerow AD, Lalezari S, Banyard D, Shaterian A, Evans GR. Lipotransfer: the potential from bench to bedside. Ann Plast Surg. 2014; 72(5):599-609.

4. Suszynski TM, Sieber DA, Van Beek AL, Cunningham BL. Characterization of adipose tissue for autologous fat grafting. Aesthet Surg J. 2015;35(2):194-203.

5. Coleman SR. Structural fat grafting: more than a permanent filler. Plast Reconstr Surg. 2006;118(3 Suppl):108S-20S.

6. Zhu M, Cohen SR, Hicok KC, Shanahan RK, Strem BM, Yu JC, et al. Comparison of three different fat graft preparation methods: gravity separation, centrifugation, and simultaneous washing with filtration in a closed system. Plast Reconstr Surg. 2013;131(4):873-80.

7. Eto $H$, Kato $H$, Suga $H$, Aoi N, Doi K, Kuno $S$, et al. The fate of adipocytes after nonvascularized fat grafting: evidence of early death and replacement of adipocytes. Plast Reconstr Surg. 2012;129(5):1081-92.

8. Liu L, Gao J, Yuan Y, Chang Q, Liao Y, Lu F. Hypoxia preconditioned human adipose derived mesenchymal stem cells enhance angiogenic potential via secretion of increased VEGF and bFGF. Cell Biol Int. 2013;37(6):551-60.

9. Pu LL, Coleman SR, Cui X, Ferguson RE Jr, Vasconez HC. Autologous fat grafts harvested and refined by the Coleman technique: a comparative study. Plast Reconstr Surg. 2008;122(3):932-7.

10. Kølle S-FT, Fischer-Nielsen A, Mathiasen AB, Elberg JJ, Oliveri RS, Glovinski $P V$, et al. Enrichment of autologous fat grafts with ex-vivo expanded adipose tissue-derived stem cells for graft survival: a randomised placebocontrolled trial. Lancet. 2013;382(9898):1113-20.

11. Yoshimura K, Suga H, Eto H. Adipose-derived stem/progenitor cells: roles in adipose tissue remodeling and potential use for soft tissue augmentation. Regen Med. 2009;4(2):265-73. 
12. Uysal CA, Tobita M, Hyakusoku H, Mizuno $H$. The effect of bone-marrowderived stem cells and adipose-derived stem cells on wound contraction and epithelization. Adv Wound Care (New Rochelle). 2014;3(6):405-13.

13. Gimble JM, Katz AJ, Bunnell BA. Adipose-derived stem cells for regenerative medicine. Circ Res. 2007;100(9):1249-60.

14. Jun-Jiang C, Huan-Jiu X. Vascular endothelial growth factor 165-transfected adipose-derived mesenchymal stem cells promote vascularization-assisted fat transplantation. Artif Cell Nanomed B. 2016;44(4):1141-9.

15. Han YD, Bai Y, Yan XL, Ren J, Zeng Q, Li XD, et al. Co-transplantation of exosomes derived from hypoxia-preconditioned adipose mesenchymal stem cells promotes neovascularization and graft survival in fat grafting. Biochem Biophys Res Commun. 2018;497(1):305-12.

16. Levy O, Zhao W, Mortensen L, Leblanc S, Tsang K, Fu M, et al. mRNAengineered mesenchymal stem cells for targeted delivery of interleukin-10 to sites of inflammation. Blood. 2013;122(14):e23-32.

17. Chien KR, Zangi L, Lui KO. Synthetic chemically modified mRNA (modRNA): toward a new technology platform for cardiovascular biology and medicine. Cold Spring Harb Perspect Med. 2014;5(1):a014035.

18. Lui KO, Zangi L, Chien KR. Cardiovascular regenerative therapeutics via synthetic paracrine factor modified mRNA. Stem Cell Res. 2014;13(3 Pt B): 693-704.

19. Witman N, Zhou C, Grote Beverborg N, Sahara M, Chien KR. Cardiac progenitors and paracrine mediators in cardiogenesis and heart regeneration. Semin Cell Dev Biol. 2020;100:29-51.

20. Zangi L, Lui KO, von Gise A, Ma Q, Ebina W, Ptaszek LM, et al. Modified mRNA directs the fate of heart progenitor cells and induces vascular regeneration after myocardial infarction. Nat Biotechnol. 2013;31(10):898-907.

21. Carlsson L, Clarke JC, Yen C, Gregoire F, Albery T, Billger M, et al. Biocompatible, purified VEGF-A mRNA improves cardiac function after intracardiac injection 1 week post-myocardial infarction in swine. Mol Ther Methods Clin Dev. 2018;9:330-46

22. Gan LM, Lagerstrom-Fermer M, Carlsson LG, Arfvidsson C, Egnell AC, Rudvik A, et al. Intradermal delivery of modified mRNA encoding VEGF-A in patients with type 2 diabetes. Nat Commun. 2019;10(1):871.

23. Yu Z, Witman N, Wang W, Li D, Yan B, Deng M, et al. Cell-mediated delivery of VEGF modified mRNA enhances blood vessel regeneration and ameliorates murine critical limb ischemia. J Control Release. 2019;310:103-14.

24. Kim JH, Kim WK, Sung YK, Kwack MH, Song SY, Choi JS, et al. The molecular mechanism underlying the proliferating and preconditioning effect of vitamin C on adipose-derived stem cells. Stem Cells Dev. 2014;23(12):1364-76.

25. Kim Kl, Park S, Im Gl. Osteogenic differentiation and angiogenesis with cocultured adipose-derived stromal cells and bone marrow stromal cells. Biomaterials. 2014;35(17):4792-804.

26. Nauta A, Seidel C, Deveza L, Montoro D, Grova M, Ko SH, et al. Adiposederived stromal cells overexpressing vascular endothelial growth factor accelerate mouse excisional wound healing. Mol Ther. 2013;21(2):445-55

27. Tonnard P, Verpaele A, Peeters G, Hamdi M, Cornelissen M, Declercq H. Nanofat grafting: basic research and clinical applications. Plast Reconstr Surg. 2013;132(4):1017-26.

28. Warren L, Manos PD, Ahfeldt T, Loh YH, Li H, Lau F, et al. Highly efficient reprogramming to pluripotency and directed differentiation of human cells with synthetic modified mRNA. Cell Stem Cell. 2010;7(5):618-30.

29. Richner JM, Himansu S, Dowd KA, Butler SL, Salazar V, Fox JM, et al. Modified mRNA vaccines protect against Zika virus infection. Cell. 2017; 169(1):176.

30. Zhang Y, Xiao LL, Li JX, Liu HW, Li SH, Wu YY, et al. Improved fat transplantation survival by using the conditioned medium of vascular endothelial growth factor transfected human adipose-derived stem cells. Kaohsiung J Med Sci. 2017;33(8):379-84.

31. Harris WM, Plastini M, Kappy N, Ortiz T, Chang S, Brown S, et al. Endothelia differentiated adipose-derived stem cells improvement of survival and neovascularization in fat transplantation. Aesthet Surg J. 2019;39(2):220-32.

32. Zheng $H, Y u Z$, Deng $M, C a i Y$, Wang $X$, Xu Y, et al. Fat extract improves fat graft survival via proangiogenic, anti-apoptotic and pro-proliferative activities. Stem Cell Res Ther. 2019;10(1):174.

33. Hu $Y$, Jiang $Y$, Wang $M$, Tian $W$, Wang $H$. Concentrated growth factor enhanced fat graft survival: a comparative study. Dermatol Surg. 2018;44(7):976-84.

34. Dominici M, Le Blanc K, Mueller I, Slaper-Cortenbach I, Marini F, Krause D, et al. Minimal criteria for defining multipotent mesenchymal stromal cells. The International Society for Cellular Therapy position statement. Cytotherapy. 2006;8(4):315-7.
35. Suga $H$, Eto $H$, Aoi N, Kato H, Araki J, Doi K, et al. Adipose tissue remodeling under ischemia: death of adipocytes and activation of stem/progenitor cells. Plast Reconstr Surg. 2010;126(6):1911-23.

36. Lancerotto L, Chin MS, Freniere B, Lujan-Hernandez JR, Li Q, Valderrama Vasquez A, et al. Mechanisms of action of external volume expansion devices. Plast Reconstr Surg. 2013;132(3):569-78.

37. Gir P, Brown SA, Oni G, Kashefi N, Mojallal A, Rohrich RJ. Fat grafting: evidence-based review on autologous fat harvesting, processing, reinjection, and storage. Plast Reconstr Surg. 2012;130(1):249-58.

38. Zuk PA, Zhu M, Mizuno H, Huang J, Futrell JW, Katz AJ, et al. Multilineage cells from human adipose tissue: implications for cell-based therapies. Tissue Eng. 2001;7(2):211-28.

39. Baer PC. Adipose-derived stem cells and their potential to differentiate into the epithelial lineage. Stem Cells Dev. 2011;20(10):1805-16

40. Toyserkani NM, Quaade ML, Sorensen JA. Cell-assisted lipotransfer: a systematic review of its efficacy. Aesthet Plast Surg. 2016;40(2):309-18.

41. Fu S, Luan J, Xin M, Wang Q, Xiao R, Gao Y. Fate of adipose-derived stromal vascular fraction cells after co-implantation with fat grafts: evidence of cel survival and differentiation in ischemic adipose tissue. Plast Reconstr Surg. 2013;132(2):363-73.

42. Li GC, Zhang HW, Zhao QC, Sun LI, Yang JJ, Hong L, et al. Mesenchymal stem cells promote tumor angiogenesis via the action of transforming growth factor beta1. Oncol Lett. 2016;11(2):1089-94.

43. Tang J, Wang J, Yang J, Kong X, Zheng F, Guo L, et al. Mesenchymal stem cells over-expressing SDF-1 promote angiogenesis and improve heart function in experimental myocardial infarction in rats. Eur J Cardiothorac Surg. 2009;36(4):644-50.

44. Zhu M, Dong Z, Gao J, Liao Y, Xue J, Yuan Y, et al. Adipocyte regeneration after free fat transplantation: promotion by stromal vascular fraction cells. Cell Transplant. 2015;24(1):49-62

45. Garza RM, Rennert RC, Paik KJ, Atashroo D, Chung MT, Duscher D, et al. Studies in fat grafting: part IV. Adipose-derived stromal cell gene expression in cell-assisted lipotransfer. Plast Reconstr Surg. 2015;135(4): 1045-55.

46. Trojahn Kolle SF, Oliveri RS, Glovinski PV, Elberg JJ, Fischer-Nielsen A, Drzewiecki KT. Importance of mesenchymal stem cells in autologous fat grafting: a systematic review of existing studies. J Plast Surg Hand Surg. 2012:46(2):59-68.

47. Naderi N, Combellack EJ, Griffin M, Sedaghati T, Javed M, Findlay MW, et al. The regenerative role of adipose-derived stem cells (ADSC) in plastic and reconstructive surgery. Int Wound J. 2017;14(1):112-24.

48. Grégoire C, Ritacco C, Hannon M, Seidel L, Delens L, Belle L, et al. Comparison of mesenchymal stromal cells from different origins for the treatment of graft-vs-host-disease in a humanized mouse model. Front Immunol. 2019;10:619.

49. Díaz-Herráez P, Saludas L, Pascual-Gil S, Simón-Yarza T, Abizanda G, Prósper F, et al. Transplantation of adipose-derived stem cells combined with neuregulin-microparticles promotes efficient cardiac repair in a rat myocardial infarction model. J Control Release. 2017;249:23-31.

50. He X, Zhong $X, N i$, Liu M, Liu S, Lan X. Effect of ASCs on the graft survival rates of fat particles in rabbits. J Plast Surg Hand Surg. 2013;47(1):3-7.

51. Zielins ER, Brett EA, Blackshear CP, Flacco J, Ransom RC, Longaker MT, et al. Purified adipose-derived stromal cells provide superior fat graft retention compared with unenriched stromal vascular fraction. Plast Reconstr Surg. 2017;139(4):911-4

52. Traktuev DO, Merfeld-Clauss S, Li J, Kolonin M, Arap W, Pasqualini R, et al. A population of multipotent CD34-positive adipose stromal cells share pericyte and mesenchymal surface markers, reside in a periendothelial location, and stabilize endothelial networks. Circ Res. 2008;102(1):77-85.

53. Zheng Y, Yi C, Xia W, Ding T, Zhou Z, Han Y, et al. Mesenchymal stem cells transduced by vascular endothelial growth factor gene for ischemic random skin flaps. Plast Reconstr Surg. 2008;121(1):59-69.

54. Soker S. Neuropilin in the midst of cell migration and retraction. Int J Biochem Cell Biol. 2001;33(4):433-7.

55. Nagy J, Dvorak A, Dvorak H. Vascular hyperpermeability, angiogenesis, and stroma generation. Cold Spring Harbor Perspect Med. 2012;2(2):a006544.

56. Magadum A, Kaur K, Zangi L. mRNA-based protein replacement therapy for the heart. Mol Ther. 2019;27(4):785-93.

57. Kormann M, Hasenpusch G, Aneja M, Nica G, Flemmer A, Herber-Jonat S, et al. Expression of therapeutic proteins after delivery of chemically modified mRNA in mice. Nat Biotechnol. 2011;29(2):154-7. 
58. Mandal P, Rossi D. Reprogramming human fibroblasts to pluripotency using modified mRNA. Nat Protoc. 2013;8(3):568-82.

59. Li J, Chen S, Zhao Z, Luo Y, Hou Y, Li H, et al. Effect of VEGF on inflammatory regulation, neural survival, and functional improvement in rats following a complete spinal cord transection. Front Cell Neurosci. 2017;11:381.

60. Won YW, Lee M, Kim HA, Nam K, Bull DA, Kim SW. Synergistically combined gene delivery for enhanced VEGF secretion and antiapoptosis. Mol Pharm. 2013;10(10):3676-83.

61. Verma M, Asakura Y, Murakonda BSR, Pengo T, Latroche C, Chazaud B, et al. Muscle satellite cell cross-talk with a vascular niche maintains quiescence via VEGF and notch signaling. Cell Stem Cell. 2018;23(4):530-43.

\section{Publisher's Note}

Springer Nature remains neutral with regard to jurisdictional claims in published maps and institutional affiliations.

Ready to submit your research? Choose BMC and benefit from:

- fast, convenient online submission

- thorough peer review by experienced researchers in your field

- rapid publication on acceptance

- support for research data, including large and complex data types

- gold Open Access which fosters wider collaboration and increased citations

- maximum visibility for your research: over $100 \mathrm{M}$ website views per year

At BMC, research is always in progress.

Learn more biomedcentral.com/submissions 